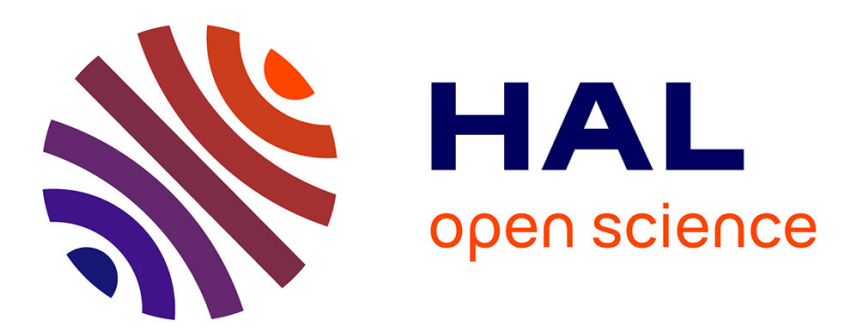

\title{
A non-linear model for the dynamics of open cross-section thin-walled beams-Part I: formulation
}

Angelo Di Egidio, Angelo Luongo, Fabrizio Vestroni

\section{To cite this version:}

Angelo Di Egidio, Angelo Luongo, Fabrizio Vestroni. A non-linear model for the dynamics of open cross-section thin-walled beams-Part I: formulation. International Journal of Non-Linear Mechanics, 2003, 38 (7), pp.1067-1081. hal-00798001

\section{HAL Id: hal-00798001 https://hal.science/hal-00798001}

Submitted on 7 Mar 2013

HAL is a multi-disciplinary open access archive for the deposit and dissemination of scientific research documents, whether they are published or not. The documents may come from teaching and research institutions in France or abroad, or from public or private research centers.
L'archive ouverte pluridisciplinaire HAL, est destinée au dépôt et à la diffusion de documents scientifiques de niveau recherche, publiés ou non, émanant des établissements d'enseignement et de recherche français ou étrangers, des laboratoires publics ou privés. 


\title{
A non-linear model for the dynamics of open cross-section thin-walled beams - Part I: formulation
}

\author{
Angelo Di Egidio ${ }^{\mathrm{a}, *}$, Angelo Luongo ${ }^{\mathrm{a}}$, Fabrizio Vestroni ${ }^{\mathrm{b}}$ \\ ${ }^{a}$ Dipartimento di Ingegneria delle Strutture, Universitá di L'Aquila, delle Acque e del Terreno, Italy \\ 'Dipartimento di Ingegneria Strutturale e Geotecnica, Universitá di Roma "La Sapienza", Italy
}

\begin{abstract}
A non-linear one-dimensional model of inextensional, shear undeformable, thin-walled beam with an open cross-section is developed. Non-linear in-plane and out-of-plane warping and torsional elongation effects are included in the model. By using the Vlasov kinematical hypotheses, together with the assumption that the cross-section is undeformable in its own plane, the non-linear warping is described in terms of the flexural and torsional curvatures. Due to the internal constraints, the displacement field depends on three components only, two transversal translations of the shear center and the torsional rotation. Three non-linear differential equations of motion up to the third order are derived using the Hamilton principle. Taking into account the order of magnitude of the various terms, the equations are simplified and the importance of each contribution is discussed. The effect of symmetry properties is also outlined. Finally, a discrete form of the equations is given, which is used in Part II to study dynamic coupling phenomena in conditions of internal resonance.
\end{abstract}

Keywords: Beams; Open cross-section; Flexural-torsional dynamics; Non-linear resonances; Warping non-linear effects

\section{Introduction}

Many papers have been devoted to non-linear dynamics of beams and recently interest has been mainly focused on non-linear motions in three dimensions. A one-dimensional polar model of a compact beam was initially studied in [1]. Since the torsional frequency of the beam is much higher than the bending ones, the torsional component was statically condensed; moreover, warping was neglected. A three-dimensional beam model was developed in [2] for a compact cross-section beam by also taking into account the warping. However, the equations of motion were truncated at the second order and only linear warping was considered.

\footnotetext{
* Corresponding author.
}

When the beam cross-section has a high aspect ratio, bending and torsional frequencies are of the same order and several internal resonance conditions can occur, involving two or three displacement components. In [3] a non-linear shear-undeformable beam model with a compact cross-section is developed, capable of studying the flexural-flexural-torsional-extensional dynamic of beam-like structures. The strain energy of the three-dimensional model is written directly in terms of the generalized deformation quantities of the polar beam model. In the energy expression, only the linear warping contribution is considered and a coupling term between torsion and axial extension is introduced. In [4-6] shear and axially undeformable beams are analyzed. In [4] flexural-torsional free motions are studied for a cantilever beam, having close bending and torsional frequencies; although beams 
with non-compact cross-section are considered, the warping effects are neglected. In [5,6] a non-linear one-dimensional polar model of compact beam is derived, capable of studying interactions between flexural and torsional motions occurring in beam-like structures in several internal resonance conditions. Even if the linear warping contribution is considered, attention is again paid to the case of compact cross-sections.

In this paper, a non-linear beam model is developed as an internally constrained three-dimensional continuum, suitable to study three dimensional large amplitude oscillations. By focusing attention on open cross-sections without any assumption of its symmetry, the effects of the torsional curvature on the elongation of the longitudinal fibers and the non-linear torsional warping of the section are considered. The warping is expressed in terms of the displacements of the shear center of the section by extending the Vlasov theory [7] to the non-linear field. This is similar to the approach found in some papers devoted to non-linear theory of a thin-walled beam [8-10]. In particular, in [10], where a moderate rotation theory of thin-walled composite beams is proposed, the series expansion of the rotation tensor has been truncated at the second order only. The beam considered here is shear and axially undeformable; these internal constraints lead to a model whose deformed configuration is described by two displacements only plus the torsional rotation. The equations of motion are derived by the Hamilton principle; they simplify remarkably if the cross-section has one or two symmetry axes. By estimating the order of magnitude of the various terms and retaining only the leading ones, simpler reduced equations are drawn. This is an extension of the model proposed in [11] for the study of the interaction between torsional and axial motions with comparable frequencies. Finally, by applying the Galerkin procedure, a discrete form of the equations of motion is obtained, in view of studying non-linear dynamic coupling phenomena dealt with in Part II [12].

\section{Kinematics}

\subsection{Displacement field}

An initially straight thin-walled beam with an open cross-section, arbitrary restrained at the ends, is considered (Fig. 1). The following hypotheses are assumed:

(H.1): the beam cross-section is rigid and remains orthogonal to the centroid axis in the deformed configuration (shear indeformable beam);

(H.2): a non-rigid displacement field is superimposed to the previous one, having components both normal and tangential to the cross-section in the deformed configuration (non-linear warping);

(H.3): (a) the shear strains on the middle surface of the thin-walled beam identically vanish (Vlasov condition); moreover, (b) the extensional and shear strains of the cross-section plane also vanish (indeformability of the section in its own plane);

(H.4): the beam is axially inextensible.

A reference frame $O x_{1} x_{2} x_{3}$ is introduced, where $x_{1}$ and $x_{2}$ are section principal axes, $x_{3}$ contains the centroid axis and $O$ is the centroid of an end cross-section (Fig. 1). A unit base vector $b=\left\{\vec{b}_{1}, \vec{b}_{2}, \vec{b}_{3}\right\}$, solid with the (not warped) section in the deformed configuration is considered, with $\vec{b}_{3}$ tangent to the centroid axis. Let us denote $\underline{b}=\left\{\underline{\vec{b}}_{1}, \underline{\vec{b}}_{2}, \underline{\vec{b}}_{3}\right\}$ as the triad solid with the section in the undeformed configuration, oriented like the $x_{i}$-axes. The displacement vector $\vec{u}_{P}=\overrightarrow{O P}-\vec{O} \underline{P}$ of the generic point $P \equiv\left(x_{1}, x_{2}, x_{3}\right)$ can be expressed as the sum of a rigid and a non-rigid displacement namely:

$$
\begin{aligned}
\vec{u}_{P}= & \vec{u}_{C}+(\Re-\mathfrak{I})\left[\left(x_{1}-x_{1_{C}}\right) \underline{\vec{b}_{1}}+\left(x_{2}-x_{2_{C}}\right) \underline{\vec{b}_{2}}\right] \\
& +\Re \sum_{i=1}^{3} \phi_{i} \underline{\vec{b}}_{i} .
\end{aligned}
$$

In Eq. (1) $\mathfrak{R}$ is the rotation tensor $\left(\vec{b}_{i}=\mathfrak{R} \vec{b}_{i}\right)$, $\mathfrak{I}$ the identity tensor, $C \equiv\left(x_{1_{C}}, x_{2_{C}}\right)$ the shear center and $\vec{\phi}=\sum_{i=1}^{3} \phi_{i} \vec{b}_{i}$ the warping vector, whose components are measured in the deformed configuration. In matrix form Eq. (1) reads as

$\mathbf{u}_{P}=\mathbf{u}_{C}+(\mathbf{R}-\mathbf{I})\left(\mathbf{x}-\mathbf{x}_{C}\right)+\mathbf{R} \phi$,

where $\mathbf{u}=\left\{\begin{array}{lll}u_{1} & u_{2} & u_{3}\end{array}\right\}^{\mathrm{T}}, \mathbf{x}=\left\{\begin{array}{lll}x_{1} & x_{2} & 0\end{array}\right\}^{\mathrm{T}}$ and $\phi=\left\{\begin{array}{lll}\phi_{1} & \phi_{2} & \phi_{3}\end{array}\right\}^{\mathrm{T}}$. To make the kinematical description unique, the warping vector must describe neither a translation nor a rotation. This requirement is satisfied if the following orthogonality conditions 


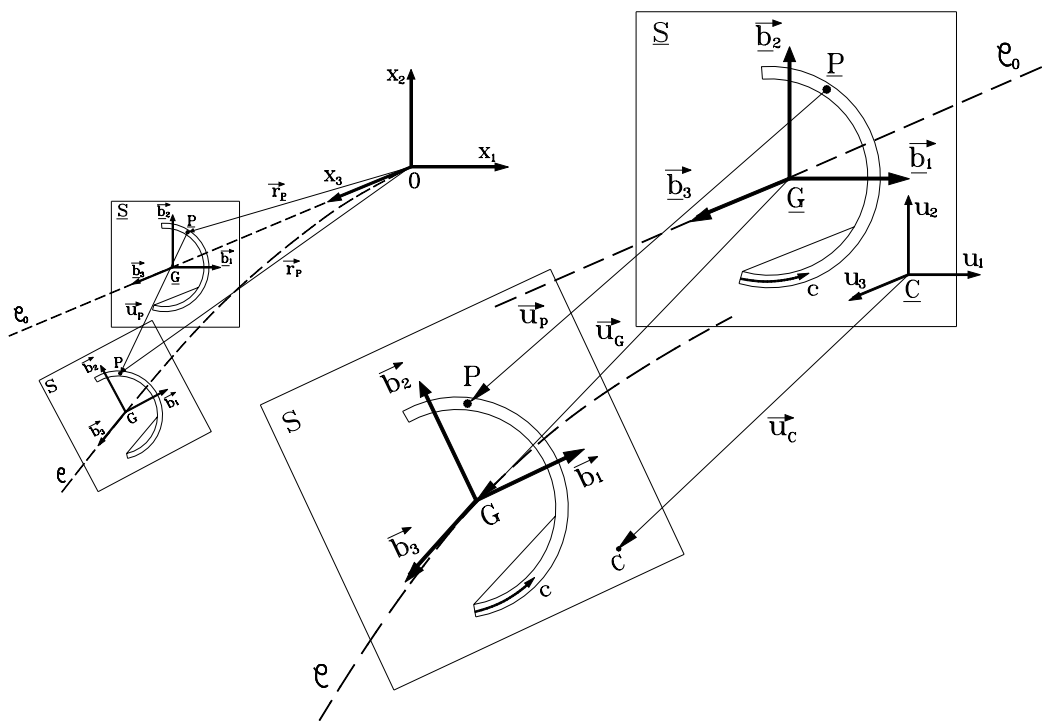

Fig. 1. Beam section before and after deformation, and unit vector triads.

hold:

$$
\begin{aligned}
& \int_{A} \phi_{3} \mathrm{~d} A=0, \quad \int_{A} \phi_{3} x_{1} \mathrm{~d} A=0, \quad \int_{A} \phi_{3} x_{2} \mathrm{~d} A=0, \\
& \int_{A} \phi_{1} \mathrm{~d} A=0, \quad \int_{A} \phi_{2} \mathrm{~d} A=0, \\
& \oint\left[\phi_{2}\left(x_{1}-x_{1_{C}}\right)-\phi_{1}\left(x_{2}-x_{2 C}\right)\right] \mathrm{d} A=0 .
\end{aligned}
$$

Eqs. $(3)_{1},(3)_{4}$ and (3) $)_{5}$ ensure $\phi$ is not a translation, Eqs. (3) $)_{2}$ and (3) 3 assure $\phi$ is not a rotation for any $\phi_{3} \neq 0$, Eq. (3) $)_{6}$ prevents $\phi$ from being a purely torsional rotation. Eqs. $(3)_{1}-(3)_{3}$ coincide with the Vlasov linear conditions, even though $\phi$ will be found here to be a non-linear function of the displacement components.

The orthogonal rotation matrix $\mathbf{R}$ describes the position of the base $b$ with respect to the base $\underline{b}(\mathbf{b}=\mathbf{R} \underline{\mathbf{b}})$. The matrix $\mathbf{R}$ is obtained through three successive elementary finite rotations around three different axes and is expressed as $\mathbf{R}=\mathbf{R}_{1} \mathbf{R}_{2} \mathbf{R}_{3}$. As illustrated in Fig. 2, $\mathbf{R}_{1}$ describes the first rotation $\vartheta_{1}$ around the axis $\underline{b}_{1}, \mathbf{R}_{2}$ describes the second rotation $\vartheta_{2}$ around the axis $\vec{b}_{2}^{\prime}$ and $\mathbf{R}_{3}$ describes the third rotation $\vartheta_{3}$ around the axis $\vec{b}_{3}^{\prime \prime} \equiv \vec{b}_{3}$. The terms of the $i$ th column of $\mathbf{R}$ are the components of the unit vector $\vec{b}_{i}$ with respect to the base $\underline{b}\left(\underline{\mathbf{b}}=\mathbf{R}^{\mathrm{T}} \mathbf{b}\right)$.

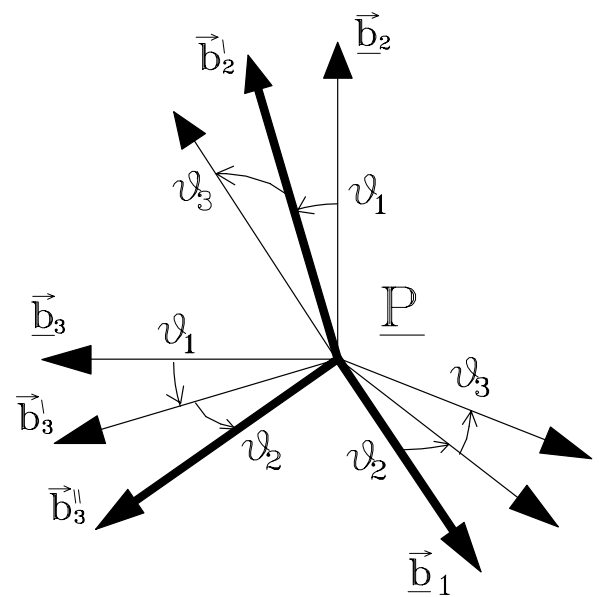

Fig. 2. Rotational sequences used to describe the orientation of the cross-section axes.

The displacements field (2) is described by six functions of the abscissa $z:=x_{3}$ and of the time $t$, i.e. $u_{i}(z, t)$ and $\vartheta_{i}(z, t)(i=1,2,3)$, and by the three warping functions $\phi_{i}\left(x_{1}, x_{2}, z, t\right)$. However, the shear indeformability condition (hypothesis H.1) makes it possible to express the flexural rotations $\vartheta_{1}$ and $\vartheta_{2}$ in terms of the spatial derivatives of the displacements $u_{i}$, thus reducing the number of independent displacement variables. 


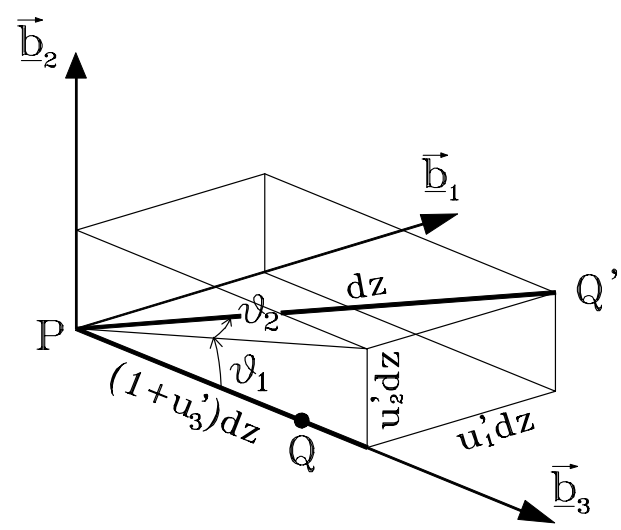

Fig. 3. Shear indeformability: geometrical interpretation.

From Fig. 3 the following relationships are drawn:

$\operatorname{tg} \vartheta_{1}=-\frac{u_{2}^{\prime}}{1+u_{3}^{\prime}}, \quad \operatorname{tg} \vartheta_{2}=\frac{u_{1}^{\prime}}{\sqrt{u_{1}^{\prime 2}+\left(1+u_{3}^{\prime}\right)^{2}}}$.

Hypotheses H.3 and H.4 will be used in the sequel. It will be shown that they permit to eliminate the warping components $\phi_{i}$ and the longitudinal displacement $u_{3}$, respectively, thus reducing to three $\left(u_{1}, u_{2}, \vartheta_{3}\right)$ the number of independent variables.

\subsection{Curvature and angular velocity}

Before analyzing the strain field, the curvatures of the beam are first defined. The curvature matrix $\mathbf{C}$, referred to the undeformed base $\underline{b}$ is

$\mathbf{C}=\mathbf{R}^{\mathrm{T}} \mathbf{R}^{\prime}$

where $(\cdot)^{\prime}=\partial / \partial z$. The result is an antisymmetric matrix whose independent components are

$$
\begin{aligned}
& \mu_{1}=\cos \vartheta_{2} \cos \vartheta_{3} \vartheta_{1}^{\prime}+\sin \vartheta_{3} \vartheta_{2}^{\prime}, \\
& \mu_{2}=-\sin \vartheta_{3} \cos \vartheta_{2} \vartheta_{1}^{\prime}+\cos \vartheta_{3} \vartheta_{2}^{\prime}, \\
& \mu_{3}=\sin \vartheta_{2} \vartheta_{1}^{\prime}+\vartheta_{3}^{\prime} .
\end{aligned}
$$

$\mu_{1}$ and $\mu_{2}$ will be referred to as flexural curvatures and $\mu_{3}$ as torsional curvature.

The angular velocity matrix $\mathbf{W}$, referred to the undeformed base $\underline{b}$, is also an antisymmetric matrix and it is given by a similar relation

$$
\mathbf{W}=\mathbf{R}^{\mathrm{T}} \dot{\mathbf{R}},
$$

where the dot denotes time differentiation. Its scalar components are

$$
\begin{aligned}
& \omega_{1}=\cos \vartheta_{2} \cos \vartheta_{3} \dot{\vartheta}_{1}+\sin \vartheta_{3} \dot{\vartheta}_{2}, \\
& \omega_{2}=-\sin \vartheta_{3} \cos \vartheta_{2} \dot{\vartheta}_{1}+\cos \vartheta_{3} \dot{\vartheta}_{2}, \\
& \omega_{3}=\sin \vartheta_{2} \dot{\vartheta}_{1}+\dot{\vartheta}_{3} .
\end{aligned}
$$

\subsection{Strain field}

The Green-Lagrange strain matrix $\mathbf{E}=\left[\varepsilon_{i j}\right]$ is assumed as the deformation measure. It is defined by

$\mathrm{d} s^{2}-\mathrm{d} \underline{s}^{2}=2 \mathrm{~d} \mathbf{x}^{\mathrm{T}} \mathrm{d} \mathbf{u}+\mathrm{d} \mathbf{u}^{\mathrm{T}} \mathrm{d} \mathbf{u}=: 2 \mathrm{~d} \mathbf{x}^{\mathrm{T}} \mathbf{E} \mathrm{d} \boldsymbol{x}$,

where $\mathrm{d} s$ and $\mathrm{d} \underline{s}$ are the deformed and undeformed length of a material segment, respectively. By differentiating Eq. (2) and substituting it into Eq. (9), the strain components $\varepsilon_{i j}$ are drawn in terms of the derivatives of the displacements $u_{i}(z, t), \vartheta_{i}(z, t)$ and $\phi_{i}\left(x_{1}, x_{2}, z, t\right)$. However, taking into account the shear-indeformability and after considerable algebra, it is possible to show (see Appendix A) that the strains $\varepsilon_{i j}$ can be expressed as a function of the curvatures $\mu_{i}$ and of the elongation $e_{C}=(\mathrm{d} s / \mathrm{d} s-1)$ of the shear-center axis, i.e. of the generalized strain measures of the one-dimensional polar beam model, in addition to the derivatives of the warping functions. The strains assume the following expressions:

$$
\begin{aligned}
{\left[\begin{array}{ll}
\varepsilon_{11} & \varepsilon_{12} \\
\varepsilon_{21} & \varepsilon_{22}
\end{array}\right]=} & {\left[\begin{array}{cc}
\phi_{1,1} & 1 / 2\left(\phi_{1,2}+\phi_{2,1}\right) \\
1 / 2\left(\phi_{2,1}+\phi_{1,2}\right) & \phi_{2,2}
\end{array}\right] } \\
& +\frac{1}{2}\left[\begin{array}{cc}
\phi_{3,1}^{2} & \phi_{3,1} \phi_{3,2} \\
\phi_{3,2} \phi_{3,1} & \phi_{3,2}^{2}
\end{array}\right]+O\left(\phi_{i}^{2}\right), \\
\left(\begin{array}{l}
\gamma_{13} \\
\gamma_{23}
\end{array}\right)= & \left(\begin{array}{c}
-\mu_{3}\left(x_{2}-x_{2_{C}}\right) \\
\mu_{3}\left(x_{1}-x_{1_{C}}\right)
\end{array}\right)+\left(1+e_{C}\right)\left(\begin{array}{l}
\phi_{3,1} \\
\phi_{3,2}
\end{array}\right) \\
& +\left(\begin{array}{c}
\phi_{1,3} \\
\phi_{2,3}
\end{array}\right)+\left[\mu_{1}\left(x_{2}-x_{2_{C}}\right)\right. \\
& \left.-\mu_{2}\left(x_{1}-x_{1_{C}}\right)+\phi_{3,3}\right]\left(\begin{array}{c}
\phi_{3,1} \\
\phi_{3,2}
\end{array}\right) \\
& +\phi_{3}\left(\begin{array}{c}
\mu_{2} \\
-\mu_{1}
\end{array}\right)+O\left(\phi_{i}^{2}, \mu_{3} \phi_{i}\right),
\end{aligned}
$$




$$
\begin{aligned}
\varepsilon_{33}= & {\left[e_{C}+\mu_{1}\left(x_{2}-x_{2_{C}}\right)-\mu_{2}\left(x_{1}-x_{1_{C}}\right)+\phi_{3,3}\right] } \\
& +\frac{1}{2}\left[e_{C}+\mu_{1}\left(x_{2}-x_{2_{C}}\right)-\mu_{2}\left(x_{1}-x_{1_{C}}\right)+\phi_{3,3}\right]^{2} \\
& +\frac{1}{2} \mu_{3}^{2}\left[\left(x_{1}-x_{1_{C}}\right)^{2}+\left(x_{2}-x_{2_{C}}\right)^{2}\right] \\
& -\mu_{3}\left[\mu_{1}\left(x_{1}-x_{1_{C}}\right)+\mu_{2}\left(x_{2}-x_{2_{C}}\right)\right] \phi_{3}+O\left(\mu_{j} \phi_{i}\right),
\end{aligned}
$$

where $i=1,2, e_{C}=u_{3}^{\prime}+1 / 2\left(u_{1}^{\prime 2}+u_{2}^{\prime 2}\right)$ and $\gamma_{i j}=$ $2 \varepsilon_{i j}$. Terms of higher order, depending on the in-plane warping $\phi_{i}$, have not been made explicit in Eqs. (10) since they will be found to be unessential for further analysis; indeed $\phi_{i}=O\left(\phi_{3}^{2}\right)(i=1,2)$, follows from Eq. $(10)_{1}$ and hypothesis H.3b. If the warping effects are neglected, Eqs. (10) becomes formally equal to the strain displacement relationships of the linear beam theory; however, here the curvatures $\mu_{i}$ and the elongation $e_{C}$ are non-linear functions of the displacements. Moreover, a new term proportional to the squared torsional curvature $\mu_{3}$ appears in the longitudinal strain $\varepsilon_{33}$; it represents the non-linear elongation of the longitudinal fibers of the beam due to the torsional rotation of the section. Finally, the in-plane strains only depend on warping, since the cross-section is rigid in bending and torsion.

Some approximations are introduced to simplify relations (10). Since the second square bracketed term in the longitudinal strain $\varepsilon_{33}$ is the square of the first term, it is neglected. Moreover, cubic terms of the kind $\mu_{i} \mu_{3} \phi_{3},(i=1,2)$ are neglected too since they are of the same order of $\mu_{3} \phi_{i}$. Finally, the elongation $e_{C}$ in the $\gamma_{13}, \gamma_{23}$ components is also neglected with respect to unity. Therefore, the approximated Green-Lagrangian strains read as

$$
\begin{aligned}
\varepsilon_{11}= & \phi_{1,1}+1 / 2 \phi_{3,1}^{2}, \\
\varepsilon_{22}= & \phi_{2,2}+1 / 2 \phi_{3,2}^{2}, \\
\gamma_{12}= & \phi_{1,2}+\phi_{2,1}+\phi_{3,1} \phi_{3,2}, \\
\gamma_{13}= & -\mu_{3}\left(x_{2}-x_{2_{C}}\right)+\phi_{3,1}+\phi_{1,3}+\left[\mu_{1}\left(x_{2}-x_{2_{C}}\right)\right. \\
& \left.-\mu_{2}\left(x_{1}-x_{1_{C}}\right)+\phi_{3,3}\right] \phi_{3,1}+\phi_{3} \mu_{2}, \\
\gamma_{23}= & \mu_{3}\left(x_{1}-x_{1_{C}}\right)+\phi_{3,2}+\phi_{2,3}+\left[\mu_{1}\left(x_{2}-x_{2_{C}}\right)\right. \\
& \left.-\mu_{2}\left(x_{1}-x_{1_{C}}\right)+\phi_{3,3}\right] \phi_{3,2}-\phi_{3} \mu_{1}, \\
\varepsilon_{33}= & e_{C}+\mu_{1}\left(x_{2}-x_{2_{C}}\right)-\mu_{2}\left(x_{1}-x_{1_{C}}\right)+\phi_{3,3} \\
& +\frac{1}{2} \mu_{3}^{2}\left[\left(x_{1}-x_{1_{C}}\right)^{2}+\left(x_{2}-x_{2_{C}}\right)^{2}\right] .
\end{aligned}
$$

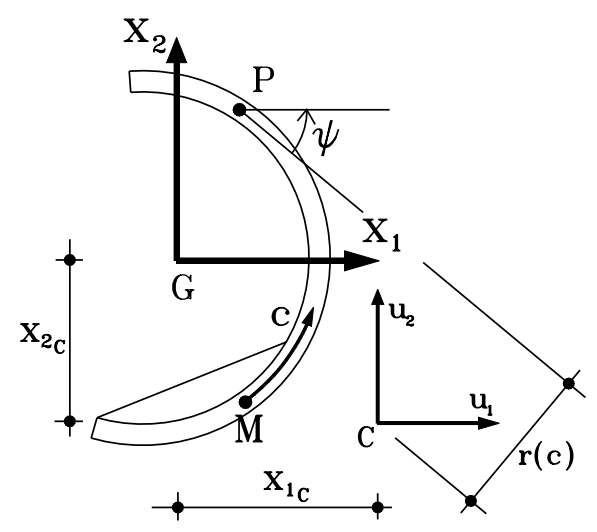

Fig. 4. Local abscissa $c$ and shear center position $C$.

\subsection{Non-linear warping functions}

In order to obtain a one-dimensional model, the dependence of the warping functions $\phi_{i}\left(x_{1}, x_{2}, z, t\right)$ on the transversal coordinates $x_{1}$ and $x_{2}$, should be determined in advance by enforcing suitable kinematical conditions. In the Vlasov linear theory the unique warping component $\phi_{3}\left(x_{1}, x_{2}, z, t\right)$ is approximated by a function $\phi_{3}^{*}(c, z, t)$ (Fig. 4) where $c$ is a curvilinear abscissa along the middle line of the section, under the hypothesis that $\phi_{3}$ is constant along the (small) thickness. Moreover, the dependence of $\phi_{3}^{*}$ on $c$ is determined by requiring that the shear strain along the middle line of the section vanishes. Here, in the non-linear problem, the same Vlasov assumption is considered (hypothesis H.3a). In addition, in order to evaluate the in-plane warping components $\phi_{1}$ and $\phi_{2}$, the in-plane strains $\varepsilon_{11}, \varepsilon_{22}$ and $\gamma_{12}$ are required to vanish on the whole section (hypothesis H.3b). In general no function having this property exists, since the plane strain problem is overdetermined; however, as shown in Appendix B, such functions $\phi_{i}$ do exist if $\phi_{3} \cong \phi_{3}^{*}$ is constant along the thickness (i.e. if the beam is thin walled) and can themselves be considered constant along the thickness, i.e. $\phi_{1} \cong \phi_{1}^{*}$ and $\phi_{2} \cong \phi_{2}^{*}$.

To evaluate the warping functions $\phi_{k}(k=1,2,3)$ (stars are omitted), the strains $\gamma_{3 c}, \varepsilon_{c c}, \varepsilon_{n n}, \gamma_{c n}$ are first evaluated, where $n$ is the (inward) normal to the middle line at $P$. By using the known rules $\gamma_{3 c}=\gamma_{13} \cos \psi+$ $\gamma_{23} \sin \psi, \varepsilon_{c c}=\varepsilon_{11} \cos ^{2} \psi+\varepsilon_{22} \sin ^{2} \psi+\gamma_{12} \cos \psi \sin \psi$ (and similar ones), where $\psi$ is the slope of the tangent to the middle line at $P$ (Fig. 4), and enforcing the 
kinematical conditions H.3, the following equations are derived:

$$
\begin{aligned}
\gamma_{3 c}= & \frac{\partial \phi_{3}}{\partial c}-\mu_{3} r+\left(\phi_{1,3} \cos \psi+\phi_{2,3} \sin \psi\right) \\
& +\phi_{3}\left(\mu_{2} \cos \psi-\mu_{1} \sin \psi\right) \\
& +\frac{\partial \phi_{3}}{\partial c}\left[\mu_{1}\left(x_{2}-x_{2_{C}}\right)-\mu_{2}\left(x_{1}-x_{1_{C}}\right)+\phi_{3,3}\right] \\
= & 0, \\
\varepsilon_{c c}= & \frac{\partial \phi_{1}}{\partial c} \cos \psi+\frac{\partial \phi_{2}}{\partial c} \sin \psi+\frac{1}{2}\left(\frac{\partial \phi_{3}}{\partial c}\right)^{2}=0, \\
\gamma_{c n}= & -\frac{\partial \phi_{1}}{\partial c} \sin \psi+\frac{\partial \phi_{2}}{\partial c} \cos \psi=0 .
\end{aligned}
$$

In Eq. $(12)_{1} x_{1}=x_{1}(c)$ and $x_{2}=x_{2}(c)$ are the parametric equations of middle line and $r=r(c)$ is the distance from the shear center $C$ of the tangent to the middle line at $P$ (Fig. 4). In Eq. $(12)_{3} \partial \phi_{3} / \partial n=0$ has been accounted for; finally, the condition $\varepsilon_{n n}=0$ is identically satisfied, since $\partial \phi_{1} / \partial n=\partial \phi_{2} / \partial n=0$. It is worth noting that Eqs. $(12)_{2}$ and $(12)_{3}$ are formally similar to that governing the linear kinematic problem of the solid line undergoing an extensional distorsion (e.g. termal variation ) $\varepsilon_{c c}=-1 / 2\left(\partial \phi_{3} / \partial c\right)^{2}$; in this context, the emergence of the in-plane warping is recognized as a consequence of the occurrence of the out-of-plane warping. Most importantly, Eqs. (12) show that $\phi_{1}$ and $\phi_{2}$ are second-order variables with respect to $\phi_{3}$, thus justifying the omission of their square in Eqs. $(11)_{4}-(11)_{6}$.

Previous remarks suggest a perturbation approach to the solution of Eqs.(12). Since the curvature $\mu_{i}$ are small, a perturbation parameter $\varepsilon$ is introduced through the ordering $\mu_{i}=\varepsilon \tilde{\mu}_{i}, \tilde{\mu}_{i}=O(1)$. Then, the warping functions are expanded as $\phi_{3}=\varepsilon \phi_{31}+\varepsilon^{2} \phi_{32}+$ $O\left(\varepsilon^{3}\right)$ and, consequently $\phi_{j}=\varepsilon^{2} \phi_{j 2}+O\left(\varepsilon^{3}\right),(j=1,2)$. The following pertubation equations up to $\varepsilon^{2}$-order are obtained (tilde omitted):

Order $\varepsilon: \quad \frac{\partial \phi_{31}}{\partial c}=\mu_{3} r$

Order $\varepsilon^{2}: \quad \frac{\partial \phi_{12}}{\partial c} \cos \psi+\frac{\partial \phi_{22}}{\partial c} \sin \psi=-\frac{1}{2}\left(\frac{\partial \phi_{31}}{\partial c}\right)^{2}$,

$$
\frac{\partial \phi_{22}}{\partial_{c}} \cos \psi-\frac{\partial \phi_{12}}{\partial_{c}} \sin \psi=0,
$$

$$
\begin{aligned}
\frac{\partial \phi_{32}}{\partial c}= & \left(\phi_{12,3} \cos \psi+\phi_{22,3} \sin \psi\right) \\
& -\phi_{31}\left(\mu_{2} \cos \psi-\mu_{1} \sin \psi\right), \\
& -\frac{\partial \phi_{31}}{\partial c}\left[\mu_{1}\left(x_{2}-x_{2_{C}}\right)\right. \\
& \left.-\mu_{2}\left(x_{1}-x_{1_{C}}\right)+\phi_{31,3}\right] .
\end{aligned}
$$

By integrating in sequence the previous equations, $\phi_{31}, \phi_{j 2}(j=1,2)$ and $\phi_{32}$ are evaluated; then, by absorbing the parameter $\varepsilon$, the following perturbation solution is drawn:

$$
\begin{aligned}
\phi_{1}= & \alpha_{7} \mu_{3}^{2}+\xi-\Theta\left(x_{2}-x_{2_{C}}\right), \\
\phi_{2}= & \alpha_{8} \mu_{3}^{2}+\eta-\Theta\left(x_{1}-x_{1_{C}}\right), \\
\phi_{3}= & \alpha_{1} \mu_{3}+\left(\alpha_{2}-\alpha_{3}\right) \mu_{1} \mu_{3}+\left(\alpha_{4}-\alpha_{5}\right) \mu_{2} \mu_{3} \\
& +\alpha_{6} \mu_{3} \mu_{3}^{\prime}-\alpha_{9} \Theta^{\prime}+\zeta_{1}\left[1+\mu_{1}\left(x_{2}-x_{2_{M}}\right)\right. \\
& \left.-\mu_{2}\left(x_{1}-x_{1_{M}}\right)\right]-\mu_{3} \zeta_{1}^{\prime} \alpha_{1} \\
& +\zeta_{2}+\xi^{\prime}\left(x_{1}-x_{1_{M}}\right)+\eta\left(x_{2}-x_{2_{M}}\right) .
\end{aligned}
$$

In Eqs. (14) 1,2 a rigid rotation has been added to the solution of Eqs. (13) 1,2 , that otherwise would be lost because of the approximations introduced (see Appendix B). In Eqs. (14) $\alpha_{i}=\alpha_{i}(c)(i=1, \ldots, 9)$ are warping functions, depending on the cross-section shape; they are not all independent and are defined to within a constant (see Appendix C). Moreover, $M$ is the principal origin of the sectorial area (Fig. 4) [7] and $\xi=\xi(z, t), \eta=\eta(z, t), \zeta_{1}(z, t), \zeta_{2}(z, t)$ and $\Theta(z, t)$ are integration arbitrary functions of $z$ and $t$. Since $\alpha_{1}$ coincides with the sectorial area, the linear part of $\phi$ is formally identical to that of the linear theory; however, here $\mu_{3}(z, t)$ represents a non-linear torsional curvature.

As shown in Appendix C, warping is described by $\alpha_{1}$ and six independent warping functions, namely $\beta_{i}=$ $\beta_{i}(c)(i=1, \ldots, 6)$, so that Eq. (14) is rewritten as

$$
\begin{aligned}
\phi_{1}= & \beta_{4} \mu_{3}^{2}+\xi(z)-\Theta\left(x_{2}-x_{2_{C}}\right), \\
\phi_{2}= & \beta_{5} \mu_{3}^{2}+\eta(z)+\Theta\left(x_{1}-x_{1_{C}}\right), \\
4 p t] \phi_{3}= & \alpha_{1} \mu_{3}\left(1-\zeta_{1}^{\prime}\right)+\beta_{1} \mu_{1} \mu_{3}+\beta_{2} \mu_{2} \mu_{3}+\beta_{3} \mu_{3}^{\prime} \mu_{3} \\
& -\beta_{6} \Theta^{\prime}+\xi^{\prime} x_{1}+\eta^{\prime} x_{2} \\
& +\zeta_{1}\left(\mu_{1} x_{2}-\mu_{2} x_{1}\right)+\tilde{\zeta}_{2},
\end{aligned}
$$


where $\tilde{\zeta}_{2}=-\xi^{\prime} x_{1_{M}}-\eta^{\prime} x_{2_{M}}-\zeta_{1} \mu_{1} x_{2_{M}}+\zeta_{1} \mu_{2} x_{1_{M}}+\zeta_{1}+\zeta_{2}$. By imposing the conditions given by Eqs. (3) $1,(3)_{4}$ and $(3)_{5}, \tilde{\zeta}_{2}, \xi, \eta$ are obtained together with the arbitrary constants; Eqs. $(3)_{2}$ and $(3)_{3}$ allows determination of $\zeta_{1} \mu_{1}$ and $\zeta_{1} \mu_{2}$; Eq. (3) $)_{6}$ determines $\Theta$. By neglecting $\zeta_{1}^{\prime}$ with respect to unity, the following final form of the warping is obtained:

$$
\begin{aligned}
& \phi_{1}=\tilde{\beta}_{4} \mu_{3}^{2}, \\
& \phi_{2}=\tilde{\beta}_{5} \mu_{3}^{2}, \\
& \phi_{3}=\alpha_{1} \mu_{3}+\tilde{\beta}_{1} \mu_{1} \mu_{3}+\tilde{\beta}_{2} \mu_{2} \mu_{3}+\tilde{\beta}_{3} \mu_{3}^{\prime} \mu_{3},
\end{aligned}
$$

where the new functions $\tilde{\beta}_{i}$ are defined in Appendix C.

By replacing Eqs. (16) in Eq. (11)6, the only non-vanishing strain $\varepsilon_{33}$ is determined

$$
\begin{aligned}
\varepsilon_{33}= & e_{G}+\mu_{1} x_{2}-\mu_{2} x_{1}+\mu_{3}^{\prime} \alpha_{1}+\frac{1}{2} \mu_{3}^{2} s^{2} \\
& +\left(\mu_{1} \mu_{3}\right)^{\prime} \tilde{\beta}_{1}+\left(\mu_{2} \mu_{3}\right)^{\prime} \tilde{\beta}_{2}+\left(\mu_{3} \mu_{3}^{\prime}\right)^{\prime} \tilde{\beta}_{3},
\end{aligned}
$$

where $e_{G}=e_{C}-\mu_{1} x_{2_{C}}+\mu_{2} x_{1_{C}}$ is the longitudinal strain of the centroid axis and $s^{2}$ is the square of the distance between the shear center of the section and the generic point $P$. The first four terms of Eq. (17) are formally equal to those of the Vlasov linear theory, with non-linear flexural and torsional curvatures. The fifth term describes the elongation due to torsion, and the remaining terms account for non-linear warping. When the beam undergoes no torsional curvature, all the linear and non-linear warping terms vanish.

\subsection{Inextensibility condition}

The beam is assumed to be inextensible (hypothesis H.4). In compact beam theory, such a property is modeled by requiring the generic element of the centroid axis to maintain its initial length. However, this condition seems to be inadequate to describe the behavior of a thin-walled beam, due to the presence of warping and torsional elongation, which in principle both induce longitudinal deformation of the centroid axis. Therefore, the inextensibility condition is introduced by requiring the mean value on the cross-section of the longitudinal strain (17) to vanish, i.e.

$\int_{A} \varepsilon_{33} \mathrm{~d} A=0$.

By taking into account that the warping functions $\alpha_{1}$ and $\beta_{i}$ satisfy Eq. (3) $)_{1}$, from Eq. (18) it follows that $e_{G}=-\frac{1}{2} \mu_{3}^{2} \rho_{C}^{2}$, where $\rho_{C}$ is the cross-section polar radius of inertia with respect to the shear center $C$.

Eq. (19) shows that the strain of the centroid axis is not zero, but it is a second-order quantity. It is not affected by warping, but only by the torsional strains, and represents the so-called non-linear torsional shortening of the beam. With Eq. (19), Eq. (17) reads as

$\varepsilon_{33}=\varepsilon_{f}+\varepsilon_{t}+\varepsilon_{\phi}^{(1)}+\varepsilon_{\phi}^{(2)}$,

where

$\varepsilon_{f}=\mu_{1} x_{2}-\mu_{2} x_{1}, \quad \varepsilon_{t}=\frac{1}{2} \mu_{3}^{2}\left(s^{2}-\rho_{C}^{2}\right)$,

$\varepsilon_{\phi}^{(1)}=\mu_{3}^{\prime} \alpha_{1}$,

$\varepsilon_{\phi}^{(2)}=\left(\mu_{1} \mu_{3}\right)^{\prime} \tilde{\beta}_{1}+\left(\mu_{2} \mu_{3}\right)^{\prime} \tilde{\beta}_{2}+\left(\mu_{3} \mu_{3}^{\prime}\right)^{\prime} \tilde{\beta}_{3}$

are the flexural, torsional, first- and second-order warping longitudinal strains, respectively. The use of Eq. (19) permits the elimination of the $u_{3}$-variable; by recalling the definition of $e_{G}$, Eq. (19) is written as

$u_{3}^{\prime}+\frac{1}{2}\left(u_{1}^{\prime 2}+u_{2}^{\prime 2}\right)-\mu_{1} x_{2_{C}}+\mu_{2} x_{1_{C}}+\frac{1}{2} \mu_{3}^{2} \rho_{C}^{2}=0$,

where $\mu_{i}=\mu_{i}\left(u_{1}, u_{2}, u_{3}, \vartheta_{3}, t\right)$, after having used the shear indeformability conditions (4). Eq. (22) is a non-linear algebraic equation in the unknown $u_{3}^{\prime}$, which is solved by a perturbation method. By introducing the ordering $u_{1}=\varepsilon \tilde{u}_{1}, u_{2}=\varepsilon \tilde{u}_{2}, \vartheta_{3}=\varepsilon \tilde{\vartheta}_{3}$, and expanding the unknown as $u_{3}=\varepsilon u_{3}^{(1)}+\varepsilon^{2} u_{3}^{(2)}+\varepsilon^{3} u_{3}^{(3)}$, the following perturbation equations are obtained up to the $\varepsilon^{2}$-order (by omitting the tilde):

$$
\begin{aligned}
& u_{3}^{\prime(1)}-\mu_{1}^{(1)} x_{2_{C}}+\mu_{2}^{(1)} x_{1_{C}}=0, \\
& u_{3}^{\prime(2)}+\frac{1}{2}\left(u_{1}^{\prime 2}+u_{2}^{\prime 2}\right)-\mu_{1}^{(2)} x_{2_{C}}+\mu_{2}^{(2)} x_{1_{C}} \\
& \quad+\frac{1}{2} \mu_{3}^{(1)^{2}} \rho_{C}^{2}=0, \\
& u_{3}^{\prime(3)}-\mu_{1}^{(3)} x_{2_{C}}+\mu_{2}^{(3)} x_{1_{C}}+\mu_{3}^{(1)} \mu_{3}^{(2)} \rho_{C}^{2}=0,
\end{aligned}
$$

where $\mu_{i}^{(k)}$ is the $\varepsilon^{k}$-order part of $\mu_{i}$. By solving the sequence of Eqs. (23), the terms of the series expansion of $u_{3}^{\prime}$ are calculated. Finally, by absorbing the perturbation parameter $\varepsilon$, a solution of the type

$u_{3}^{\prime}=f\left(u_{1}, u_{2}, \vartheta_{3}, t\right)$

is obtained, not shown here for purpose of brevity [13]. This internal constraint, valid for thin-walled beams, is more complicated than that for compact beams. 
However, it reduces to the simplest one if the cross-section has two symmetry axes and the torsional elongation effects are neglected.

\section{Equations of motion}

\subsection{Order-three equations}

The equations of motion of the thin-walled beam are obtained via the generalized Hamilton principle. Since approximated order-three equations are sought, order-three expansions of the kinematic relationships are directly used in the functional. It reads as

$$
\begin{aligned}
\delta H= & \int_{t_{1}}^{t_{2}} \int_{0}^{l}\left\{\delta L\left(u_{1}, u_{2}, u_{3}, \vartheta_{3}, t\right)+Q_{1} \delta u_{1}\right. \\
& +Q_{2} \delta u_{2}+Q_{\vartheta} \delta \vartheta_{3} \\
& +\delta\left[\lambda\left(f\left(u_{1}, u_{2}, \vartheta_{3}, t\right)-u_{3}^{\prime}\right]\right\} \mathrm{d} z \mathrm{~d} t \\
& \forall\left(\delta u_{1}, \delta u_{2}, \delta u_{3}, \delta \vartheta_{3}\right),
\end{aligned}
$$

where $l$ is the length of the beam, $L=T-U$ is the Lagrangian for unit length, $T$ is the kinetic energy and $U$ is the elastic potential energy; moreover, $Q$ 's are generalized distributed non-conservative forces spending work on the associated virtual displacements and $\lambda$ is a Lagrangian multiplier associated with the inextensibility constraint (24). This latter has to be taken into account in the variational principle since the velocity $\dot{u}_{3}$, which appears in the kinetic energy $T$, cannot be directly eliminated by means of Eq. (24). On the contrary, the constraints (4) and (24), as previously said, have already been employed to eliminate $\vartheta_{1}, \vartheta_{2}$ and $u_{3}$ in the elastic potential energy $U$.

By neglecting the warping inertia effects, the kinetic energy reads as

$$
\begin{aligned}
T= & \frac{1}{2} \int_{0}^{l} \sum_{i=1}^{3}\left(m \dot{u}_{i_{O}}^{2}+\mathfrak{I}_{i} \omega_{i}^{2}\right) \mathrm{d} z \\
\simeq & \frac{1}{2} \int_{0}^{l}\left(\sum_{i=1}^{3} m \dot{u}_{i}^{2}+m x_{2_{C}} \dot{u}_{1} \dot{\vartheta}_{3}-m x_{1_{C}} \dot{u}_{2} \dot{\vartheta}_{3}\right. \\
& \left.+\frac{1}{2} \mathfrak{I}_{C} \dot{\vartheta}_{3}^{2}\right) \mathrm{d} z,
\end{aligned}
$$

where $\dot{u}_{i_{O}}$ is the $i$ th velocity component of the centroid $O, m$ the mass per unit length of the beam, $\mathfrak{I}_{i}$ the cross-section mass-moment with respect to the $x_{i}$-axis and $\mathfrak{I}_{C}$ the polar mass-moment with respect to the shear center. In Eq. (26) use has been made of Eq. (2) (with $\phi=0$ ) and Eqs. (8). The rotation matrix $\mathbf{R}$ and the angular velocities (8) have been linearized and, as is usual in slender beams, flexural rotatory inertia terms have been neglected. It can be shown (see Section 3.2) that the order of magnitude of the terms neglected is small with respect to the retained terms.

By restricting the analysis to isotropic beams and neglecting the contribution of the Poisson ratio, the elastic potential energy per unit length reads as

$$
\begin{aligned}
V= & \frac{1}{2} \int_{A}\left[G\left(\gamma_{31}^{2}+\gamma_{32}^{2}\right)+E \varepsilon_{33}^{2}\right] \mathrm{d} A \\
= & \frac{1}{2} G J \mu_{3}^{2}+\frac{1}{2} E I_{1} \mu_{1}^{2}+\frac{1}{2} E I_{2} \mu_{2}^{2}+\frac{1}{2} E \Gamma \mu_{3}^{\prime 2} \\
& +\frac{1}{2} E \int_{A}\left[\varepsilon_{t}^{2}+\varepsilon_{\phi}^{(2)^{2}}+2\left(\varepsilon_{f} \varepsilon_{t}+\varepsilon_{f} \varepsilon_{\phi}^{(1)}+\varepsilon_{f} \varepsilon_{\phi}^{(2)}\right.\right. \\
& \left.\left.+\varepsilon_{t} \varepsilon_{\phi}^{(1)}+\varepsilon_{t} \varepsilon_{\phi}^{(2)}\right)\right] \mathrm{d} A,
\end{aligned}
$$

where $G$ and $E$ are elastic moduli, $G J$ is the St. Venant torsional stiffness, $E I_{i}$ are flexural stiffness and $E \Gamma$ the warping torsional stiffness of the linear theory. In Eq. (27), $\gamma_{31}$ and $\gamma_{32}$, (which are not zero out of the section middle line) are assumed to contribute to the St. Venant torsional elastic term only; i.e. they are approximated by the linear part of Eqs. (11) $)_{5,6}$, in which $\phi_{3}$ is taken equal to the more refined solution of the Neumann problem, which takes account of the variation along the thickness. In addition, use has been made of Eqs. (20) and (21).

By substituting Eqs. (26) and (27) in the Hamilton principle and making the variations, Eq. (25) assumes the following form:

$$
\begin{aligned}
& \int_{t_{1}}^{t_{2}} \int_{0}^{l}\left\{\sum _ { i = 1 } ^ { 2 } \left(Q_{i} \delta u_{i}+H_{i t} \delta \dot{u}_{i}+H_{i 1} \delta u_{i}^{\prime}\right.\right. \\
& \left.+H_{i 2} \delta u_{i}^{\prime \prime}+H_{i 3} \delta u_{i}^{\prime \prime \prime}+H_{i 4} \delta u_{i}^{\prime \prime \prime \prime}\right)+H_{\vartheta t} \delta \dot{\vartheta}_{3} \\
& +H_{\vartheta 0} \delta \vartheta_{3}+H_{\vartheta 1} \delta \vartheta_{3}^{\prime}+H_{\vartheta 2} \vartheta_{3}^{\prime \prime}+H_{\vartheta 3} \delta \vartheta_{3}^{\prime \prime \prime}=0 \\
& \left.\quad m \dot{u}_{3} \delta \dot{u}_{3}-\lambda \delta u_{3}^{\prime}+\left[f\left(u_{1}, u_{2}, \vartheta_{3}\right)-u_{3}^{\prime}\right) \delta \lambda\right\} \\
& \quad \times \mathrm{d} z \mathrm{~d} t=0 \\
& \quad \forall\left(\delta u_{1}, \delta u_{2}, \delta u_{3}, \delta \vartheta_{3}, \delta \lambda\right)
\end{aligned}
$$


where the functions $H_{\alpha}=H_{\alpha}\left(u_{1}, u_{2}, u_{3}, \vartheta_{3}, \lambda, t\right)$ have complicated expressions, reported in [13]. By performing integrations by parts, the following four equations are obtained from Eq. (28), in addition to Eq. (24)

$m \ddot{u}_{3}-\lambda^{\prime}=0$

and

$$
\begin{aligned}
& m_{1} \ddot{u}_{1}+m x_{2_{C}} \ddot{\vartheta}_{3}-Q_{1} \\
& \quad=-H_{11}^{\prime}+H_{12}^{\prime \prime}-H_{13}^{\prime \prime \prime}+H_{14}^{\prime \prime \prime \prime}:=G_{1}^{\prime},
\end{aligned}
$$$$
m_{2} \ddot{u}_{2}+m x_{1_{C}} \ddot{\vartheta}_{3}-Q_{2}
$$$$
=-H_{21}^{\prime}+H_{22}^{\prime \prime}-H_{23}^{\prime \prime \prime}+H_{24}^{\prime \prime \prime \prime}:=G_{2}^{\prime},
$$$$
\Im_{C} \ddot{\vartheta}_{3}+m x_{2} \ddot{u}_{1}-m x_{1} \ddot{u}_{2}-Q_{\vartheta}
$$$$
=H_{\vartheta 0}-H_{\vartheta 1}^{\prime}+H_{\vartheta 2}^{\prime \prime}-H_{\vartheta 3}^{\prime \prime \prime}=: G_{\vartheta}
$$

with the relevant boundary conditions:

$$
\begin{aligned}
& \left.\left(W_{i 1} \delta u_{i}+W_{i 2} \delta u_{i}^{\prime}+W_{i 3} \delta u_{i}^{\prime \prime}+W_{i 4} \delta u_{i}^{\prime \prime \prime}\right)\right|_{0} ^{l}=0, \\
& \left.\left(W_{\vartheta 0} \delta \vartheta_{3}+W_{\vartheta 1} \delta \vartheta_{3}^{\prime}+W_{\vartheta 2} \delta \vartheta_{3}^{\prime \prime}\right)\right|_{0} ^{l}=0,
\end{aligned}
$$

where $W_{i 1}=H_{i 1}-H_{i 2}^{\prime}+H_{i 3}^{\prime \prime}-H_{i 4}^{\prime \prime \prime}, W_{i 2}=H_{i 2}-$ $H_{i 3}^{\prime}+H_{i 4}^{\prime \prime}, W_{i 3}=H_{i 3}-H_{i 4}^{\prime}, W_{i 4}=H_{i 4}(i=1,2)$ and $W_{\vartheta 0}=H_{\vartheta 1}-H_{\vartheta 2}^{\prime}+H_{34}^{\prime \prime}, W_{\vartheta 1}=H_{\vartheta 2}-H_{\vartheta 3}^{\prime}, W_{\vartheta 2}=H_{\vartheta 3}$.

From Eq. (29) the physical meaning of $\lambda$ emerges, which represents the inertial longitudinal forces due to the assumption of the inextensibility of the beam. By integrating Eq. (29) and using Eq. (24), the Lagrangian multiplier is first evaluated in terms of the displacements:

$$
\begin{aligned}
\lambda= & \left.\int_{l}^{z} m \int_{0}^{z}\left[f\left(u_{1}, u_{2}, \vartheta_{3}\right) \mathrm{d} z+u_{3}(0)\right)\right] \cdot \mathrm{d} z \\
& +G(l)
\end{aligned}
$$

then it is substituted in Eqs. (30) and (31). A set of three integro-differential equations in the unknown $u_{1}, u_{2}, \vartheta_{3}$ is finally drawn. They constitute order-three equations of motion suitable to study flexural-torsional motions of thin-walled beams with open cross-section.

\subsection{Discussion of the equations}

When the equations of motion (30) and the relevant boundary conditions (31) are expressed in terms of the independent displacement components $u_{1}, u_{2}, \vartheta_{3}$,
Table 1

Coupling terms in the elastic potential energy

\begin{tabular}{llll}
\hline & No symmetries & One symmetry & Two symmetries \\
\hline$\varepsilon_{f} \varepsilon_{t}$ & Yes & Yes & No \\
$\varepsilon_{f} \varepsilon_{\phi}^{(2)}$ & Yes & Yes & No \\
$\varepsilon_{t} \varepsilon_{\phi}^{(1)}$ & Yes & No & No \\
$\varepsilon_{t} \varepsilon_{\phi}^{(2)}$ & Yes & Yes & Yes \\
\hline
\end{tabular}

the equations contain some hundreds of terms, such that they can be handled only by an algebraic manipulator. These equations simplify if the cross-section has one or two symmetry axes. Referring to the compact form of the elastic potential energy, Eq. (27), it is easy to check that, if symmetries are present, some coupling terms between longitudinal strains of different nature vanish. From Table 1 it is seen that, for mono-symmetric cross-sections, coupling between torsional elongation and linear warping disappears. For double symmetric cross-sections, coupling between flexure and torsional elongation and flexure and non-linear warping also vanish. However, torsional elongation and non-linear warping are always coupled.

Another type of analysis which has been performed on the equations of motion (30) and (31) consists of evaluating the order of magnitude of the various terms. The analysis is conducted with the aim to simplify the equations, by retaining only the most important terms. To this end, the equations of motion and the relevant boundary conditions are first put in non-dimensional form by introducing the following quantities:

$$
\begin{aligned}
& \tilde{u}_{1}=\frac{u_{1}}{l}, \quad \tilde{u}_{2}=\frac{u_{2}}{l}, \quad \tilde{\vartheta}_{3}=\frac{h}{l} \vartheta_{3}=\frac{\vartheta_{3}}{\rho}, \\
& \tilde{z}=\frac{z}{l}, \quad \tilde{t}=\omega t .
\end{aligned}
$$

In Eq. (33) $\omega$ is a linear frequency of the beam and $h$ is a characteristic dimension of the cross-section, introduced to render the torsion $\tilde{\vartheta}_{3}$ of the same order of the displacements $\tilde{u}_{i}$ when $u_{i}=O\left(\vartheta_{3} h\right)$. The tilde will be omitted in the sequel.

No ordering scheme is introduced on the three configuration variables, which still remain of the same order, namely $O\left(u_{1}\right)=O\left(u_{2}\right)=O\left(\vartheta_{3} h\right)=\varepsilon$, where $\varepsilon$ is a small quantity. The order of magnitude of the spatial and time derivatives of the generic configuration 


\begin{tabular}{llll}
\hline & Linear terms & Quadratic terms & Cubic terms \\
\hline$\varepsilon_{f}^{2}$ & $O(1) \varepsilon$ & $O(1) \varepsilon^{2}, O\left(h^{2} / l^{2} \pi^{2}\right) \varepsilon^{2}$ & $O(1) \varepsilon^{3}, O\left(h^{2} / l^{2} \pi^{2}\right) \varepsilon^{3}$ \\
$\varepsilon_{t}^{2}$ & - & - & $O(1) \varepsilon^{3}, O\left(h^{2} / l^{2} \pi^{2}\right) \varepsilon^{3}$ \\
$\varepsilon_{\phi}^{(1)^{2}}+\varepsilon_{\phi}^{(2)^{2}}$ & $O(1) \varepsilon$ & $O\left(h^{2} / l^{2} \pi^{2}\right) \varepsilon^{2}$ & $O\left(h^{2} / l^{2} \pi^{2}\right) \varepsilon^{3}$ \\
$\varepsilon_{t} \varepsilon_{f}$ & - & $O(1) \varepsilon^{2}$ & $O(1) \varepsilon^{3}, O\left(h^{2} / l^{2} \pi^{2}\right) \varepsilon^{3}$ \\
$\varepsilon_{t} \varepsilon_{\phi}^{(1)}$ & - & $O(1) \varepsilon^{2}$ & $O\left(h^{2} / l^{2} \pi^{2}\right) \varepsilon^{3}$ \\
$\varepsilon_{t} \varepsilon_{\phi}^{(2)}$ & - & - & $O\left(h^{2} / l^{2} \pi^{2}\right) \varepsilon^{3}$ \\
$\varepsilon_{f} \varepsilon_{\phi}^{(1)}+\varepsilon_{f} \varepsilon_{\phi}^{(2)}$ & - & $O\left(h^{2} / l^{2} \pi^{2}\right) \varepsilon^{2}$ & $O\left(h^{2} / l^{2} \pi^{2}\right) \varepsilon^{3}$ \\
\hline
\end{tabular}

variable $u(z, t)$ is evaluated according to the following rules:

$$
\frac{\partial^{n} u}{\partial t^{n}}=O(\varepsilon), \quad \frac{\partial^{n} u}{\partial z^{n}}=O\left(\pi^{n} \varepsilon\right)
$$

by assuming $u$ to be a bi-periodic function of time frequency 1 and spatial frequency $\pi$. The order of magnitude of the various contributions to the elastic potential energy (27), thus obtained, is showed in Table 2.

It is found that, among the quadratic terms, the leading order contributions are of purely flexural nature and of torsional-flexural and torsional-linear warping nature; among the cubic terms, the first two types of terms and the purely torsional elongation terms are important. Therefore, the torsional elongation and linear warping play an important role in the description of the mechanical behavior of thin-walled beams with an open cross-section, while the non-linear warping contribution is less important with respect to the previous ones. Terms depending on the Lagrangian multiplier are found to be negligible; longitudinal inertia forces and non-linear inertia contributions produce higher order effects and the relevant terms can be omitted in the kinetic energy (26). This is in accordance with [3,6] where only the linear rotational inertia contribution are considered.

After having retained only the leading order terms, the reduced equations of motion contain about 50 terms yet, if no symmetries exist.

\subsection{Discrete model}

The reduced equations of motion are discretized according to the Galerkin procedure. The independent displacements vector $\mathbf{u}=\left\{u_{1}, u_{2}, \vartheta_{3}\right\}^{\mathrm{T}}$ is expressed as a linear combination of given $z$-function vectors $\mathbf{f}_{k}(z)=$ $\left\{f_{k 1}(z), f_{k 2}(z), f_{k 3}(z)\right\}^{\mathrm{T}}$ and unknown $t$-function coefficients $q_{k}(t)$ :

$\mathbf{u}(z, t)=\sum_{k=1}^{n} q_{k}(t) \mathbf{f}_{k}(z)$.

The functions $\mathbf{f}_{k}(z)$ are chosen as eigenfunctions of the linearized equations and boundary conditions. Since for a generic cross-section even the linear equations are coupled, all the components of $\mathbf{f}_{k}(z)$ are different from zero.

From the equations of motion (30) and boundary conditions (31) the following variational dimensionless equation is drawn, where the tilde is omitted for simplicity:

$$
\begin{aligned}
& \int_{0}^{1}\left\{-G_{1} \delta u_{1}^{\prime}+\left(-\mu \ddot{u}_{1}-\rho \mu \tilde{x}_{2_{C}} \ddot{\vartheta}_{3}+Q_{1}\right) \delta u_{1}\right. \\
& \quad-G_{2} \delta u_{2}^{\prime}+\left(-\mu \ddot{u}_{2}+\rho \mu \tilde{x}_{1_{C}} \ddot{\vartheta}_{3}+Q_{2}\right) \delta u_{2} \\
& \quad+\left(G_{\vartheta}-\rho^{2} v \ddot{\vartheta}_{3}-\rho \mu \tilde{x}_{2_{C}} \ddot{u}_{1}+\rho \mu \tilde{x}_{1_{C}} \ddot{u}_{2}\right. \\
& \left.\left.\quad+Q_{\vartheta}\right) \delta \vartheta_{3}\right\} \mathrm{d} z+\left\{\left(W_{12} \delta u_{1}^{\prime}+W_{13} \delta u_{1}^{\prime \prime}+W_{14} \delta u_{1}^{\prime \prime \prime}\right)\right. \\
& \quad+\left(W_{22} \delta u_{2}^{\prime}+W_{23} \delta u_{2}^{\prime \prime}+W_{24} \delta u_{2}^{\prime \prime \prime}\right) \\
& \left.\quad+\left(W_{\vartheta 0} \delta \vartheta_{3}+W_{\vartheta 1} \delta \vartheta_{3}^{\prime}+W_{\vartheta 2} \delta \vartheta_{3}^{\prime \prime}\right)\right\}\left.\right|_{0} ^{1}=0
\end{aligned}
$$

where

$$
\begin{aligned}
& \mu=\frac{m}{G J} \omega^{2} l^{4}, \quad v=\frac{\Im_{C} \omega^{2} l^{2}}{G J}, \quad \tilde{x}_{1_{C}}=\frac{x_{1_{C}}}{l}, \\
& \tilde{x}_{2_{C}}=\frac{x_{2_{C}}}{l}
\end{aligned}
$$

are non-dimensional quantities describing translational and rotational masses and the coordinates of 
the shear center, respectively. By substituting Eqs. (35) into Eq. (36) and vanishing separately terms in $\delta q_{k}, 3 n$ ordinary differential equations of motion follow. By limiting the expansion (35) to $n=3$ terms (e.g. by assuming a group of three modes with similar wave-length), three non-linear equations of the following type are obtained:

$$
\begin{aligned}
\ddot{q}_{h}+\omega_{h}^{2} q_{h}= & \sum_{i=1}^{3} \sum_{j=1}^{3} c_{h i j} q_{i} q_{j}+\sum_{i=1}^{3} \sum_{j=1}^{3} \sum_{k=1}^{3} \\
& \times c_{h i j k} q_{i} q_{j} q_{k}+f_{h} \quad(h=1,2,3),
\end{aligned}
$$

where $\omega_{h}$ is the $h$ th linear frequency, $f_{h}$ the $h$ th modal force, and $c_{h i j}$ and $c_{h i j k}$ are coefficients depending on eigenfunctions. In the general case all quadratic and cubic terms appear in each equation of motion.

If the cross-section has a symmetry axis, e.g. $x_{1}$, Eq. (35) becomes

$$
\begin{aligned}
\mathbf{u}(z, t)= & \left(\begin{array}{c}
f_{11}(z) \\
0 \\
0
\end{array}\right) q_{1}(t)+\left(\begin{array}{c}
0 \\
f_{22}(z) \\
f_{32}(z)
\end{array}\right) q_{2}(t) \\
& +\left(\begin{array}{c}
0 \\
f_{23}(z) \\
f_{33}(z)
\end{array}\right) q_{3}(t)
\end{aligned}
$$

and the discretized equations of motion (38) read as

$$
\begin{aligned}
\ddot{q}_{1}+\omega_{1}^{2} q_{1}= & c_{1} q_{2}^{2}+c_{2} q_{3}^{2}+c_{3} q_{2} q_{3}+c_{4} q_{1} q_{2}^{2} \\
& +c_{5} q_{1} q_{3}^{2}+c_{6} q_{1} q_{2} q_{3}+f_{1} \\
\ddot{q}_{2}+\omega_{2}^{2} q_{2}= & c_{7} q_{1} q_{2}+c_{8} q_{1} q_{3}+c_{9} q_{2}^{3}+c_{10} q_{3}^{3} \\
& +c_{11} q_{1}^{2} q_{2}+c_{12} q_{1}^{2} q_{3}+c_{13} q_{2} q_{3}^{2} \\
& +c_{14} q_{2}^{2} q_{3}+f_{2} \\
\ddot{q}_{3}+\omega_{3}^{2} q_{3}= & c_{15} q_{1} q_{2}+c_{16} q_{1} q_{3}+c_{17} q_{2}^{3}+c_{18} q_{3}^{3} \\
& +c_{19} q_{1}^{2} q_{2}+c_{20} q_{1}^{2} q_{3}+c_{21} q_{2} q_{3}^{2} \\
& +c_{22} q_{2}^{2} q_{3}+f_{3} .
\end{aligned}
$$

If the cross-section has two symmetry axes, Eq. (35) is written as

$$
\mathbf{u}(z, t)=\left(\begin{array}{l}
f_{1}(z) q_{1}(t) \\
f_{2}(z) q_{2}(t) \\
f_{3}(z) q_{3}(t)
\end{array}\right)
$$

and the discretized equations of motion (38) become

$$
\begin{gathered}
\ddot{q}_{1}+\omega_{1}^{2} q_{1}=c_{1} q_{2} q_{3}+c_{2} q_{1} q_{3}^{2}+f_{1}, \\
\ddot{q}_{2}+\omega_{2}^{2} q_{2}=c_{3} q_{1} q_{3}+c_{4} q_{2} q_{3}^{2}+f_{2}, \\
\ddot{q}_{3}+\omega_{3}^{2} q_{3}=c_{5} q_{1} q_{2}+c_{6} q_{3}^{3}+c_{7} q_{1}^{2} q_{3} \\
+c_{8} q_{2}^{2} q_{3}+f_{3} .
\end{gathered}
$$

In Eqs. $(40)_{1}$ and $(42)_{1}$ the $q_{1}^{3}$-term is not present, since it is of higher order, as discussed previously. Although the case of two symmetry axes is the most similar to the case of compact cross-section beams studied in [1], in Eq. (42) $)_{3}$ the $q_{3}^{3}$ term is also present and this is due to the purely torsional elongation effect. Terms in Eqs. (38) and (40) involve many different internal resonance conditions, which will be discussed in Part II of this paper. However, it must be stressed here that the kind of non-linear terms in Eqs. (38) and (39) remains unchanged if the warping and the torsional elongation effects are omitted, so that such deformations do not introduce new internal resonances. On the contrary, they strongly affect the coefficients of Eqs. (38) and (40) and therefore are important to correctly describe the non-linear behavior of the beam.

\section{Conclusion}

A non-linear one-dimensional model of a thinwalled beam with open cross-section and shear and axially undeformable has been developed. The Green-Lagrange tensor has been adopted as a strain measure; its components depend only upon the strain quantities of the one-dimensional polar beam model, in addition to the warping. The Vlasov kinematical hypothesis, which was formulated in the linear framework, has been extended to the non-linear field; in particular, the in-plane displacements, which arise as a second-order effect of the out-of-plane classical warping, have been accounted for. The constraint of in-plane indeformability of the cross-section makes it possible to express warping in terms of the curvatures of the beam. Consequently, the independent displacement components necessary to describe the beam deformed configuration reduce to three, two transversal translations of the shear-center axis and one torsional rotation. 
Three differential equations of motion, containing quadratic and cubic non-linearities, have been obtained via the Hamilton principle. These equations are rather complex and can be handled only by an algebraic manipulator. Symmetries produce great simplifications. An analysis has been conducted to estimate the order of magnitude of the terms appearing in the equations, which made it possible to discuss the relative importance of the different contributions and to omit several terms. In particular, non-linear warping does not significantly affect the behavior of the beam, since its magnitude is small with respect to the other non-linear terms.

Finally, discrete equations have been obtained for a three-mode approximation able to describe coupling phenomena in three-dimensional motion. It has been found that even in the most general case of no-symmetry axis, warping and torsional elongation do not add any new kind of terms to the discrete equations but they affect the values of the coefficients. In particular torsional elongation produces a remarkable modification of the coefficients. Therefore, it is expected that torsional elongation, more than non-linear warping, would play an important role in the description of the responce of the thin-walled beam. The matter will be discussed in Part II [12].

\section{Acknowledgements}

This work has been partially supported by the fund of MURST (Ministry of University, Scientific and Technological Research) under the Grant COFIN 97-98, 99-00.

\section{Appendix A. Strain-displacement relationships}

With the aim to obtain the deformation field expressed in terms of the torsional and flexural curvatures, a suitable partitioning of the displacement field (2) is developed as follows:

$$
\begin{aligned}
\left(\begin{array}{c}
\mathbf{v} \\
\mathbf{w}
\end{array}\right)= & \left(\begin{array}{c}
\mathbf{v}_{C} \\
\mathbf{w}_{C}
\end{array}\right)+\left[\begin{array}{cc}
\mathbf{R}_{y y}-\mathbf{I} & \mathbf{R}_{y z} \\
\mathbf{R}_{z y} & \mathbf{R}_{z z}-\mathbf{I}
\end{array}\right]\left(\begin{array}{c}
\mathbf{y}-\mathbf{y}_{C} \\
0
\end{array}\right) \\
& +\left[\begin{array}{ll}
\mathbf{R}_{y y} & \mathbf{R}_{y z} \\
\mathbf{R}_{z y} & \mathbf{R}_{z z}
\end{array}\right]\left(\begin{array}{c}
\phi_{y} \\
\phi_{z}
\end{array}\right),
\end{aligned}
$$

where $\mathbf{v}=\left\{\begin{array}{ll}u_{1} & u_{2}\end{array}\right\}^{\mathrm{T}}$ and $\mathbf{w}=\left\{u_{3}\right\}$ are the displacement components of the point $P$ in the plane of the section and out-of-plane, respectively, $\mathbf{y}=\left\{\begin{array}{ll}x_{1} & x_{2}\end{array}\right\}^{\mathrm{T}}$ and $\mathbf{z}=\left\{x_{3}\right\}$ are the in-plane and out-of-plane coordinates of $P, \phi_{y}=\left\{\phi_{1} \phi_{2}\right\}^{\mathrm{T}}$ and $\phi_{z}=\left\{\phi_{3}\right\}$ are the in-plane and out-of-plane warping functions, $\mathbf{y}_{C}=$ $\left\{\begin{array}{ll}x_{1_{C}} & x_{2_{C}}\end{array}\right\}^{\mathrm{T}}$ are the coordinates of the shear center, $\mathbf{R}_{i j}$ are sub-matrices of matrix $\mathbf{R}$.

From Eq. (A.1), after differentiation, it follows that

$$
\begin{aligned}
\left(\begin{array}{c}
\mathrm{d} \mathbf{v} \\
\mathrm{d} \mathbf{w}
\end{array}\right)= & \left(\begin{array}{c}
{\left[\mathbf{v}_{C}^{\prime}+\mathbf{R}_{y y}^{\prime}\left(\mathbf{y}-\mathbf{y}_{C}\right)\right] \mathrm{d} \mathbf{z}+\left(\mathbf{R}_{y y}-\mathbf{I}\right) \mathrm{d} \mathbf{y}} \\
{\left[\mathbf{w}_{C}^{\prime}+\mathbf{R}_{z y}^{\prime}\left(\mathbf{y}-\mathbf{y}_{C}\right)\right] \mathrm{d} \mathbf{z}+\mathbf{R}_{z y} \mathrm{~d} \mathbf{y}}
\end{array}\right) \\
+ & \left(\begin{array}{c}
\left(\mathbf{R}_{y y} \phi_{y, y}+\mathbf{R}_{y z} \phi_{z, y}\right) \mathrm{d} \mathbf{y} \\
+\left(\mathbf{R}_{y y} \phi_{y}+\mathbf{R}_{y z} \phi_{z}\right)_{, z} \mathrm{~d} \mathbf{z} \\
\left(\mathbf{R}_{z y} \phi_{y, y}+\mathbf{R}_{z z} \phi_{z, y}\right) \mathrm{d} \mathbf{y} \\
+\left(\mathbf{R}_{z y} \phi_{y}+\mathbf{R}_{z z} \phi_{z}\right)_{, z} \mathrm{~d} \mathbf{z}
\end{array}\right)
\end{aligned}
$$

By substituting $\mathrm{d} \mathbf{u}=\{\mathrm{d} \mathbf{v} d \mathbf{w}\}^{\mathrm{T}}$ and $\mathrm{d} \mathbf{x}=\{\mathrm{d} \mathbf{y} d \mathbf{z}\}^{\mathrm{T}}$ in Eq. (9) the strain matrix $\mathbf{E}$ is obtained. Taking into account the orthogonality property of $\mathbf{R}$ (i.e. $\mathbf{R}^{\mathrm{T}} \mathbf{R}=$ $\mathbf{R} \mathbf{R}^{\mathrm{T}}=\mathbf{I}$ ) and definition (5) of the curvature matrix $\mathbf{C}$, and using the following identities proved in [13]:

$$
\begin{aligned}
& \left(\mathbf{R}_{y y}-\mathbf{R}_{y y}^{\mathrm{T}}\right)=\operatorname{skew}\left[\mathbf{R}_{y y}\right]=0, \\
& \left(\mathbf{y}-\mathbf{y}_{C}\right)^{\mathrm{T}}\left(\mathbf{R}_{y y}^{\prime \mathrm{T}} \mathbf{R}_{y y}^{\prime}+\mathbf{R}_{z y}^{\prime \mathrm{T}} \mathbf{R}_{z y}^{\prime}\right)\left(\mathbf{y}-\mathbf{y}_{C}\right) \\
& \quad=\left(\mathbf{y}-\mathbf{y}_{C}\right)^{\mathrm{T}}\left(\mathbf{C}_{y y}^{\mathrm{T}} \mathbf{C}_{y y}+\mathbf{C}_{z y}^{\mathrm{T}} \mathbf{C}_{z y}\right)\left(\mathbf{y}-\mathbf{y}_{C}\right), \\
& \left(\mathbf{R}_{y z}^{\prime \mathrm{T}} \mathbf{R}_{y y}^{\prime}+\mathbf{R}_{z z}^{\prime \mathrm{T}} \mathbf{R}_{z y}^{\prime}\right)\left(\mathbf{y}-\mathbf{y}_{C}\right)=\mathbf{C}_{y z}^{\mathrm{T}} \mathbf{C}_{y y}\left(\mathbf{y}-\mathbf{y}_{C}\right), \\
& \left(\mathbf{R}_{y z}^{\prime \mathrm{T}} \mathbf{R}_{y z}^{\prime}+\mathbf{R}_{z z}^{\prime \mathrm{T}} \mathbf{R}_{z y}^{\prime}\right)=\mathbf{C}_{y z}^{\mathrm{T}} \mathbf{C}_{y z}
\end{aligned}
$$

the components of the matrix $\mathbf{E}$ become

$$
\begin{aligned}
\mathbf{E}_{y y}= & \frac{1}{2}\left(\phi_{y, y}^{\mathrm{T}}+\phi_{y, y}\right)+\phi_{z, y}^{\mathrm{T}} \phi_{z, y}+O\left(\phi_{y}^{2}\right), \\
2 \mathbf{E}_{y z}= & {\left[\mathbf{C}_{y y}\left(\mathbf{y}-\mathbf{y}_{C}\right)\right]+\left[\mathbf{R}_{y y}^{\mathrm{T}} \mathbf{v}_{C}^{\prime}+\mathbf{R}_{z y}^{\mathrm{T}}\left(1+\mathbf{w}_{C}^{\prime}\right)\right] } \\
& +\phi_{z, y}^{\mathrm{T}}\left[\mathbf{R}_{z z}^{\mathrm{T}}\left(1-\mathbf{w}_{C}^{\prime}\right)+\mathbf{R}_{y z}^{\mathrm{T}} \mathbf{v}_{C}^{\prime}\right] \\
& +\mathbf{C}_{y z} \phi_{z}+\phi_{z, y}^{\mathrm{T}} \mathbf{C}_{z y}\left(\mathbf{y}-\mathbf{y}_{C}\right)+\phi_{z, y}^{\mathrm{T}} \phi_{z, z} \\
& +\phi_{y, z}+O\left(\phi_{y}^{2}, \mathbf{C}_{\alpha \beta} \phi_{y}\right) .
\end{aligned}
$$




$$
\begin{aligned}
\mathbf{E}_{z z}= & \left(e_{C}+1 / 2 e_{C}^{2}\right)+\left[\mathbf{v}_{C}^{\prime \mathrm{T}} \mathbf{R}_{y y}^{\prime}+\left(1+\mathbf{w}_{C}^{\prime}\right)^{\mathrm{T}} \mathbf{R}_{z y}^{\prime}\right]\left(\mathbf{y}-\mathbf{y}_{C}\right) \\
& +1 / 2\left(\mathbf{y}-\mathbf{y}_{C}\right)^{\mathrm{T}}\left(\mathbf{C}_{y y}^{\mathrm{T}} \mathbf{C}_{y y}+\mathbf{C}_{z y}^{\mathrm{T}} \mathbf{C}_{z y}\right)\left(\mathbf{y}-\mathbf{y}_{C}\right) \\
& +\left[\left(1+\mathbf{w}_{C}^{\prime}\right)^{\mathrm{T}} \mathbf{R}_{z z}^{\prime}+\mathbf{v}_{C}^{\prime \mathrm{T}} \mathbf{R}_{y z}^{\prime}\right] \phi_{z}+\mathbf{C}_{y z}^{\mathrm{T}} \mathbf{C}_{y y} \\
& \times\left(\mathbf{y}-\mathbf{y}_{C}\right) \phi_{z}+1 / 2 \mathbf{C}_{y z}^{\mathrm{T}} \mathbf{C}_{y z} \phi_{z}^{2} \\
& +\left[\left(1+\mathbf{w}_{C}^{\prime}\right)^{\mathrm{T}} \mathbf{R}_{z z}+\mathbf{v}_{C}^{\prime} \mathbf{R}_{y z}\right] \phi_{z, z} \\
& +\mathbf{C}_{z y}\left(\mathbf{y}-\mathbf{y}_{C}\right) \phi_{z, z}+1 / 2 \phi_{z, z}^{2}+O\left(\mathbf{C}_{\alpha \beta} \phi_{y}\right),
\end{aligned}
$$

warping $\phi$. By posing

$$
\mathbf{E}_{y y}=\left[\begin{array}{cc}
\varepsilon_{x x} & \varepsilon_{x y} \\
\varepsilon_{y x} & \varepsilon_{y y}
\end{array}\right], \quad 2 \mathbf{E}_{y z}=\left\{\begin{array}{l}
\gamma_{x z} \\
\gamma_{y z}
\end{array}\right\}, \quad \mathbf{E}_{z z}=\varepsilon_{z z}
$$

and recalling the definition of the curvatures $\mu_{i}$ :

$$
\mathbf{C}=\mathbf{R}^{\mathrm{T}} \mathbf{R}^{\prime}=\left[\begin{array}{ll}
\mathbf{C}_{y y} & \mathbf{C}_{y z} \\
\mathbf{C}_{z y} & \mathbf{C}_{z z}
\end{array}\right]=\left[\begin{array}{cc|c}
0 & -\mu_{3} & \mu_{2} \\
\mu_{3} & 0 & -\mu_{1} \\
\hline-\mu_{2} & \mu_{1} & 0
\end{array}\right],
$$

where $(\alpha, \beta=y, z)$ and the following relation $\left(e_{C}+\right.$ $\left.1 / 2 e_{C}^{2}\right)=\mathbf{w}_{C}^{\prime}+1 / 2\left(\mathbf{v}_{C}^{\prime 2}+\mathbf{w}_{C}^{\prime 2}\right)$ has been used, with $e_{C}$ the elongation of the shear-center axis. Terms of higher order have not been reported for sake of simplicity.

The shear indeformability condition, in matrix form, reads as

$\mathbf{t}=\left(1+e_{C}\right) \mathbf{R} \underline{\mathbf{b}}_{3}$,

where $\mathbf{t}=\left\{\begin{array}{ll}\mathbf{v}_{C}^{\prime} & 1+\mathbf{w}_{C}^{\prime}\end{array}\right\}^{\mathrm{T}}$ is a vector tangent to the shear-center axis in the actual configuration and $\underline{\mathbf{b}}_{3}=$ $\left\{\begin{array}{ll}\mathbf{0} & 1\end{array}\right\}^{\mathrm{T}}$ is the unit vector aligned with the axis in the undeformed configuration. From (A.5) it follows that

$\mathbf{v}_{C}^{\prime}=\left(1+e_{C}\right) \mathbf{R}_{y z}$,

$1+\mathbf{w}_{C}^{\prime}=\left(1+e_{C}\right) \mathbf{R}_{z z}$.

By using Eqs. (A.6), the strains (A.4) further simplify into

$$
\begin{aligned}
& \mathbf{E}_{y y}= \frac{1}{2}\left(\phi_{y, y}^{\mathrm{T}}+\phi_{y, y}\right)+\phi_{z, y}^{\mathrm{T}} \phi_{z, y}+O\left(\phi_{y}^{2}\right), \\
& 2 \mathbf{E}_{y z}= \mathbf{C}_{y y}\left(\mathbf{y}-\mathbf{y}_{C}\right)+\phi_{z, y}^{\mathrm{T}}\left[\left(1+e_{C}\right)\right. \\
&\left.+\mathbf{C}_{z y}\left(\mathbf{y}-\mathbf{y}_{C}\right)+\phi_{z, z}\right] \\
&+\mathbf{C}_{y z} \phi_{z}+\phi_{y, z}+O\left(\phi_{y}^{2}, C_{\alpha \beta} \phi_{y}\right) . \\
& \mathbf{E}_{z z}=\left(e_{C}+1 / 2 e_{C}^{2}\right)+\left(1+e_{C}\right) \mathbf{C}_{z y}\left(\mathbf{y}-\mathbf{y}_{C}\right) \\
& \quad+1 / 2\left(\mathbf{y}-\mathbf{y}_{C}\right)^{\mathrm{T}}\left(\mathbf{C}_{y y}^{\mathrm{T}} \mathbf{C}_{y y}+\mathbf{C}_{z y}^{\mathrm{T}} \mathbf{C}_{z y}\right)\left(\mathbf{y}-\mathbf{y}_{C}\right) \\
& \quad+\mathbf{C}_{y z}^{\mathrm{T}} \mathbf{C}_{y y}\left(\mathbf{y}-\mathbf{y}_{C}\right) \phi_{z}+1 / 2 \mathbf{C}_{y z}^{\mathrm{T}} \mathbf{C}_{y z} \phi_{z}^{2} \\
& \quad+\left(1+e_{C}\right) \phi_{z, z}+\mathbf{C}_{z y}\left(\mathbf{y}-\mathbf{y}_{C}\right) \phi_{z, z} \\
& \quad+1 / 2 \phi_{z, z}^{2}+O\left(\mathbf{C}_{\alpha \beta} \phi_{y}\right) .
\end{aligned}
$$

Eqs. (A.7) show that the strains depend only on the curvatures $\mathbf{C}_{i j}$, on the elongation $e_{C}$, and on the expressions (10) are finally obtained.

\section{Appendix B. The cross-section in-plane strain problem}

The cross-section in-plane indeformability condition (hypothesis H.3b), requires the strains $\varepsilon_{11}, \varepsilon_{22}$ and $\gamma_{12}$ (Eqs. $\left.(11)_{1}-(11)_{3}\right)$ identically vanish on the section, i.e. the following equations are satisfied:

$$
\begin{aligned}
& \phi_{1,1}=-\frac{1}{2} \phi_{3,1}^{2}, \\
& \phi_{2,2}=-\frac{1}{2} \phi_{3,2}^{2}, \\
& \phi_{1,2}+\phi_{2,1}=-\phi_{3,1} \phi_{3,2} .
\end{aligned}
$$

Eqs. (B.1) are a set of three linear differential equations in the two unknowns $\phi_{1}=\phi_{1}\left(x_{1}, x_{2}, z, t\right)$ and $\phi_{2}=\phi_{2}\left(x_{1}, x_{2}, z, t\right)$ in which $\phi_{3}=\phi_{3}\left(x_{1}, x_{2}, z, t\right)$ can be considered as a 'known' term. It constitutes the classical plane strain problem of the linear continuum mechanics. Since the problem is overdetermined, it generally does not admit solution, unless the compatibility equation $\left(\phi_{1,1}\right)_{, 22}+\left(\phi_{2,2}\right)_{, 11}=\left(\phi_{1,2}+\phi_{2,1}\right)_{, 12}$ is satisfied. For problem (B.1) it reads as

$$
\left|\begin{array}{ll}
\phi_{3,11} & \phi_{3,12} \\
\phi_{3,12} & \phi_{3,22}
\end{array}\right|=0
$$

i.e., it requires that the Hessian of the out-of-plane warping $\phi_{3}$ identically vanishes in the section. Therefore, from a geometrical point of view, condition (B.2) demands one of the two principal curvature of the warped section is everywhere zero. It is worth noticing that such a condition is not satisfied by the solution of the Neuman problem $\nabla^{2} \phi_{3}=0$, since it entails $\phi_{3,11} \phi_{3,22}<0$. In contrast, it is satisfied if, according to Vlasov, $\phi_{3}$ is assumed constant along the (small) 
thickness of section, since one of its principal curvatures vanishes in each point.

To solve Eqs. (B.1) it is convenient to resort to curvilinear coordinates $(c, n)$, with $c$ a curvilinear abscissa along the cross-section middle line and $n$ the (inward) normal distance along the chord. The change of coordinates reads as

$x_{1}(c, n)=x_{1}^{0}(c)-n \sin \psi(c)$,

$x_{2}(c, n)=x_{2}^{0}(c)+n \cos \psi(c)$,

where $\left(x_{1}^{0}(c), x_{2}^{0}(c)\right)$ are the coordinates of a point $P^{0}$ on the middle line and $\psi(c)$ the slope of the tangent at $P^{0}$. By rotating the strain tensor in the new base and expressing its components $\left(\varepsilon_{c c}, \varepsilon_{n n}\right.$ and $\left.\gamma_{c n}\right)$ in the new coordinates $(c, n)$, the undeformability conditions (B.1) transform as follows:

$$
\begin{aligned}
\varepsilon_{c c}:= & \frac{1}{1-n / R}\left(\phi_{1, c} \cos \psi(c)+\phi_{2, c} \sin \psi(c)\right) \\
& +\frac{1}{2}\left(\phi_{3, c}\right)^{2}=0,
\end{aligned}
$$$$
\varepsilon_{n n}:=-\phi_{1, n} \sin \psi(c)+\phi_{2, n} \cos \psi(c)+\frac{1}{2}\left(\phi_{3, n}\right)^{2}
$$$$
=0 \text {, }
$$

$\gamma_{c n}:=\frac{1}{1-n / R}\left(\phi_{2, c} \cos \psi(c)-\phi_{1, c} \sin \psi(c)\right)$

$$
+\left(\phi_{1, n} \cos \psi(c)-\phi_{2, n} \sin \psi(c)\right)+\phi_{3, c} \phi_{3, n}
$$

$$
=0 \text {, }
$$

where $R:=(\mathrm{d} \psi / \mathrm{d} c)^{-1}$ is the curvature radius of the middle line of the section at $P^{0}$. If $\phi_{3}$ is constant along the thickness, then $\phi_{3, n} \equiv 0$ and Eqs. (B.4) $)_{2,3}$ become homogeneous; at the same order of approximation, even $\phi_{1}$ and $\phi_{2}$ can be assumed constant, so that $\varepsilon_{n n}=0$ is identically satisfied. Finally, by neglecting $n / R$ with respect to the unity, Eqs. $(12)_{2}$ and $(12)_{3}$ are obtained, from which $\phi_{1}(c, z, t)$ and $\phi_{2}(c, z, t)$ are drawn as functions of the 'known' component $\phi_{3}(c, z, t)$. It can be observed that $\phi_{1}$ and $\phi_{2}$ are found to within a rigid motion (see Eqs. $(14)_{2}$ and $(14)_{3}$ ); this latter is an exact solution for the complete Eqs. (B.4), but not for the approximated Eqs. $(12)_{2}$ and (12) $)_{3}$.

\section{Appendix C. Non-linear warping functions}

Warping functions in Eq. (14) are

$$
\begin{aligned}
\alpha_{1}(c)= & \int_{0}^{c} r(c) \mathrm{d} c \\
\alpha_{2}(c)= & \int_{0}^{c} \alpha_{1}(c) \sin \psi(c) \mathrm{d} c+\bar{\alpha}_{2}, \\
\alpha_{3}(c)= & \int_{0}^{c} \alpha_{1}^{\prime}(c)\left(x_{2}(c)-x_{2_{c}}\right) \mathrm{d} c+\bar{\alpha}_{3}, \\
\alpha_{4}(c)= & \int_{0}^{c} \alpha_{1}^{\prime}(c)\left(x_{1}(c)-x_{1_{C}}\right) \mathrm{d} c+\bar{\alpha}_{4}, \\
\alpha_{5}(c)= & \int_{0}^{c} \alpha_{1}(c) \cos \psi(c) \mathrm{d} c+\bar{\alpha}_{5}, \\
\alpha_{6}(c)= & \int_{0}^{c}\left[\alpha_{1}(c) r(c)+2 \alpha_{7}(c) \cos \psi(c)\right. \\
& \left.+2 \alpha_{8}(c) \sin \psi(c)\right] \mathrm{d} c+\bar{\alpha}_{6}, \\
\alpha_{7}(c)= & -\frac{1}{2} \int_{0}^{c} r^{2}(c) \cos \psi(c) \mathrm{d} c+\bar{\alpha}_{7}, \\
\alpha_{8}(c)= & -\frac{1}{2} \int_{0}^{c} r^{2}(c) \sin \psi(c) \mathrm{d} c+\bar{\alpha}_{8}, \\
\alpha_{9}(c)= & \int_{0}^{c}\left[x_{2}(c) \cos \psi(c)\right. \\
& \left.-x_{1}(c) \sin \psi(c)\right] \mathrm{d} c+\bar{\alpha}_{9} .
\end{aligned}
$$

The independent non-linear shape warping functions in Eq. (15) are

$$
\begin{aligned}
\beta_{1}(c) & =\alpha_{2}(c)-\alpha_{3}(c) \\
& =-2 \alpha_{3}(c)+\alpha_{1}(c)\left(x_{2}(c)-x_{2_{C}}\right)+\bar{\beta}_{1}, \\
\beta_{2}(c) & =\alpha_{4}(c)-\alpha_{5}(c) \\
& =2 \alpha_{4}(c)-\alpha_{1}(c)\left(x_{1}(c)-x_{1_{c}}\right)+\bar{\beta}_{2}, \\
\beta_{3}(c) & =\alpha_{6}(c)+\bar{\beta}_{3}, \quad \beta_{4}(c)=\alpha_{7}(c)+\bar{\beta}_{4}, \\
\beta_{5}(c) & =\alpha_{8}(c)+\bar{\beta}_{5}, \quad \beta_{6}(c)=\alpha_{9}(c)+\bar{\beta}_{6},
\end{aligned}
$$

where $\bar{\alpha}_{i}$ are integration constants and $\bar{\beta}_{1}=\bar{\alpha}_{2}-$ $\bar{\alpha}_{3}, \bar{\beta}_{2}=\bar{\alpha}_{4}-\bar{\alpha}_{5}, \bar{\beta}_{3,4,5,6}=\bar{\alpha}_{6,7,8,9}$. The warping 
functions in Eq. (16) are defined as

$$
\begin{aligned}
& \tilde{\beta}_{i}=\beta_{i}^{*}-\bar{\beta}_{i}-k_{i 1} x_{1}-k_{i 2} x_{2}, \quad i=(1,2,6), \\
& \tilde{\beta}_{3}=\beta_{3}^{*}-\bar{\beta}_{3}-k_{31} x_{1}-k_{32} x_{2}-2 \tilde{\beta}_{6} k_{45}, \\
& \tilde{\beta}_{4}=\beta_{4}^{*}-\bar{\beta}_{4}-k_{45} x_{2}, \\
& \tilde{\beta}_{5}=\beta_{5}^{*}-\bar{\beta}_{5}+k_{45} x_{1},
\end{aligned}
$$

where $\beta_{j}^{*}$ are functions (C.2) in which the constants $\bar{\beta}_{j}=0$. The quantities $\bar{\beta}_{j}, k_{i 1}, k_{i 2}, k_{45},(j=1, \ldots, 6)(i=$ $1,2,3,6)$ are

$$
\begin{array}{ll}
\bar{\beta}_{j}=\frac{\int_{A} \beta_{j}^{*} \mathrm{~d} A}{A}, \quad k_{i 1}=\frac{\int_{A} \beta_{i}^{*} x_{1} \mathrm{~d} A}{I_{2}}, & k_{i 2}=\frac{\int_{A} \beta_{i}^{*} x_{2} \mathrm{~d} A}{I_{1}}, \\
k_{45}=\frac{\int_{A}\left(\beta_{5}^{*} x_{1}-\beta_{4}^{*} x_{2}\right) \mathrm{d} A}{\left(I_{1}+I_{2}\right)}, & \text { (C.4) }
\end{array}
$$

\section{References}

[1] M.R.M. Crespo da Silva, C.C. Glynn, Nonlinear flexural-flexural-torsional dynamics of inextensional beams. I. Equations of motion, J. Struct. Mech. 6 (4) (1978) 437-448.

[2] A. Rosen, P. Friedmann, The nonlinear behavior of elastic slender straight beams undergoing small strain and moderate rotations, J. Appl. Mech. 46 (1979) 161-168.

[3] M.R.M. Crespo da Silva, Non-linear flexural-flexuraltorsional-extensional dynamics of beams-I. Formulation, Int. J. Solids Struct. 24 (12) (1988) 1225-1234.
[4] A. Luongo, G. Rega, F. Vestroni, Non resonant non-planar free motions of inextensional non-compact beams, J. Sound Vib. 134 (1) (1989) 73-86.

[5] M.R.M. Crespo da Silva, Equations for nonlinear analysis of 3D motions of beams, Appl. Mech. Rev. 44 (11 part 2) (1991) S51-S59.

[6] M.R.M. Crespo da Silva, C.L. Zaretzky, Nonlinear flexural-flexural-torsional interactions in beams including the effect of torsional dynamics. I: Primary resonance, Nonlinear Dynamics 5 (1994) 3-23.

[7] V.Z. Vlasov, Thin-Walled Elastic Beams, Monson, Jerusalem, 1961.

[8] A.A. Ghobarah, W.K. Tso, A non-linear thin-walled beam theory, Internat. J. Mech. Sci. 13 (1971) 1025-1033.

[9] H. Mollmann, Finite displacements of thin-walled beams. Part 1, geometrical foundations, Report no. 252, Danish Center for Appl. Math. and Mech., Technical University of Denmark, 1982, pp. 1-48.

[10] L. Ascione, L. Feo, On the mechanical behaviour of thin-walled beams of open cross-section, Int. J. Engng. Analysis Design 2 (1995) 14-34.

[11] A. Di Egidio, A. Luongo, F. Vestroni, A nonlinear model of open cross section beams for dynamic interaction analysis, EUROMECH-2nd European Nonlinear Oscillation Conference, Prague, September 9-13, 1996.

[12] A. Di Egidio, A. Luongo, F. Vestroni, A non-linear model for the dynamics of open cross-section thin-walled beamsPart II: forced motion, Int. J. Non-Linear Mech. 37 (2003) 1083-1094.

[13] A. Di Egidio, A nonlinear model for beams with an open cross section for the study of 3D large amplitude oscillations, Ph.D Thesis, Universitá degli Studi di Roma "La Sapienza", 1997 (in Italian). 